\title{
Endoscopically Guided Stapled Gastric Fundectomy in Treatment of Bleeding Fundal Varices
}

\author{
El-Sheikh M, MD; Hossam I, MD; Abdel Hadey H, MD; Jsmail T, MD.
}

\author{
Department of General Surgery, Tanta University, Egypt
}

\begin{abstract}
Aim: To evaluate endoscopically guided stapled gastricfundectomy with peri-esophagogastric devascularization and splenectomy in treatment of bleeding fundal varices.

Patients and methods: This study was performed on 24 patients (18 males and 6 females) with bleeding gastric varices hospitalized for either bleeding gastric fundal varices or previous episodes of variceal bleeding. Gastric fundectomy and periesophagogastric devascularization was undertaken. The site of fundal varices was determined with intraoperative endoscopy, Application of non-crushing curved intestinal clamp until the disappearance of fundal varices was done. After disappearance of fundal varices as noted by the endoscope, resection of the fundus bearing varices at this site of clamping was done using linear stapler for anastomosis. Over sewing of the staple line was done. The mean length of follow up was $49.3 \pm 10.2$ months

Results: Mean age was $48.6 \pm 12.6$ years. Patients had $2.42 \pm 1.56$ bleeding attacks. Endoscopic sclerotherapy succeeded in 20 patients (83.33\%), 4 patients (16.67\%) were treated with balloon tamponade and in 2 patients (8.33\%), operation was performed for uncontrollable bleeding. 21 patients (87.50\%) belonged to A, 2 patients $(8.33 \%)$ were $B$ while the remaining 1 patient (8.33\%) was C Child-Pugh scoring. He was operated for uncontrollable hematemesia. 14 patients (58.33\%) had isolated gastric varices and 10 patients $(41.47 \%)$ had gastroesophageal varices. 11 patients (33.33\%) had positive serum hepatitis C virus antibody. The patient with C Child-Pugh scoring deceased due to hepatocellular failure. Seven patients (29.16\%) experienced one or more early postoperative complications: 3 wound sepsis, 2 small subphrenic collections, 2 pleural effusions, 1 bronchitis, and 1 portal vein thrombosis. One patient developed delayed gastric emptying. One patient developed incisional hernia. There was no bleeding from gastric varices and no endoscopic recurrences. Three patients had 1- 3 attacks of hematemesis from esophageal varices that was successfully controlled by sclerotherapy. 3 patients developed malignancies: 2 hepatocellular carcinomas and 1 uterine cancer. Four patients died of hepatic failure, including the 2 with hepatocellular carcinomas. By univariate analysis, age, Child Pugh scoring and absence of malignancy were significantly associated with survival. Postoperative downgrading of Child-Pugh scoring of patients was noticed. Mean hemoglobin level at the time of admission was $7.99 \pm 1.35 \mathrm{~g} / \mathrm{dL}$. By the third month, it reached $11.90 \pm 0.89 \mathrm{~g} / \mathrm{dL}$ ( $\mathrm{p}$ less than 0.0001).

Conclusions: Gastric fundectomy and periesophagogastric devascularization is associated with a low incidence of recurrent bleeding and offers good long-term survival. It is an effective therapeutic intervention in patients with fundal variceal bleeding. Intra-operative endoscopy helps to guide accurate localization and complete eradication of varices and to minimize the resected area of the gastric fundus.
\end{abstract}

\section{Introduction:}

Gastric variceal bleeding in cirrhotic patients is a life-threatening situation. The mortality rate of gastric variceal bleeding in cirrhotic patients has been reported to be $45-55 \%{ }^{1}$ Gastric varices may occur at the fundus, the lesser curvature, or at both, and when fundal varices are the origin of 
hemorrhage, the risk of death may be as high as $78 \%$ during the first 6 months following non-operative treatments like endoscopy. ${ }^{2}$

According to $\operatorname{Sarin}^{3}$ when the gastroesophageal varices (GOV) are an extension of esophageal varices, they are categorized into two types. The most common are Type 1 gastroesophageal varices (GOV1), which extend along the lesser curvature. Type 2 gastroesophageal varices (GOV2) are those which extend along the fundus. Isolated gastric varices (IGV) occur in the absence of esophageal varices and are also classified into two types. Type 1 (IGV1) are located in the fundus, and type 2 (IVG2) are located in the body, antrum, or around the pylorus.

Sarin's classification is useful for considering the management of gastric varices. Bleeding from the GOV1 is controlable with endoscopic injection sclerotherapy or endoscopic variceal ligation, while it is still hard to control bleeding from fundic gastric varices, such as GOV2 and IGV1. ${ }^{3}$

The options for management of gastric variceal hemorrhage are: Endoscopic sclerotherapy, but it has been reported that traditional endoscopic injection sclerotherapy is ineffective for the treatment of isolated gastric varices, $, 4,5$ Balloonoccluded retrograde transvenous obliteration (B-RTO); for the treatment fundic gastric varices. ${ }^{6}$ Transjugular portosystemic shunt (TIPS); may be used as a salvage treatment with failed endoscopic management during attack of bleeding 7,8 or surgical; in the form of devascularization of the upper stomach with splenectomy. ${ }^{9}$

Most of the studies showed that massive gastric variceal bleeding is less amenable to endoscopic intervention. Furthermore, recurrent variceal bleeding occurs in $30 \%-$ $50 \%$ of patients, even after successful nonsurgical intervention. ${ }^{10}$

Hans etal,(2004), performed a combination of peri-esophagogastric devascularization, splenectomy and fundectomy. The extent of fundal resection was limited to the engorged varix bearing area. The extent of the resection was determined by palpation under direct vision after the gastric wall was opened. This operative method was effective in controlling fundal variceal bleeding. In addition, no recurrent gastric bleeding has been encountered among patients that survived this operation. ${ }^{11}$

El-Gengehy M., et al, (2007) reported that to achieve control of gastric variceal hemorrhage, fundal mini-gasterectomy and splenectomy (for IGV 1) and with added devascularization of the gastro-esophageal junction (for GOV 2), could be considered a good alternative operative procedure. Endoscopic follow up proved that all fundal varices disappeared completely with downgrading of esophageal varices. ${ }^{12}$

At present, the most satisfactory indicator to detect the presence and localization of fundal varices is the endoscope. Intraoperative Endoscopic examination helps to localize varices to determine the part of fundus to be resected.

The aim of the work was to evaluate endoscopically guided stapled minigasterectomy with peri-esophagogastric devascularization and splenectomy in treatment of bleeding fundal varices.

\section{Patients and methods:}

This study was performed in General Surgery Department, Tanta University Hospitals, Egypt during the period from January 2009 to December 2013 on 24 patients with bleeding gastric varices. Full explanation of procedures; possible complications and patient consent were assured before inclusion in the research. The study protocol was approved by the Ethics Committee of General Surgery Department, Tanta University Hospitals.

Our study included patients with one or more of the following inclusion criteria: Isolated gastric varices in the fundus (IGV) or Gastro-esophageal varices (GOV) with history of hematemesis or during an attack of hematemesis that was uncontrollable by nonoperative means.

Patients with any of the following criteria were excluded from our study: Isolated gastric varices in the lesser curvature or cardia or gastroesophageal varices with gastric varices 


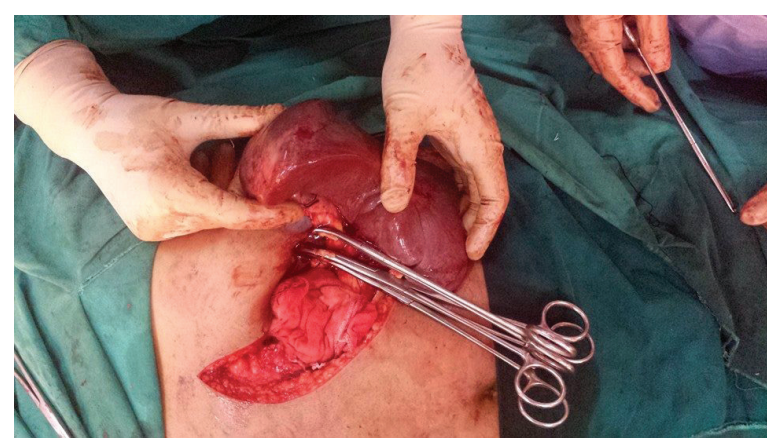

Figure (1): Splenectomy.

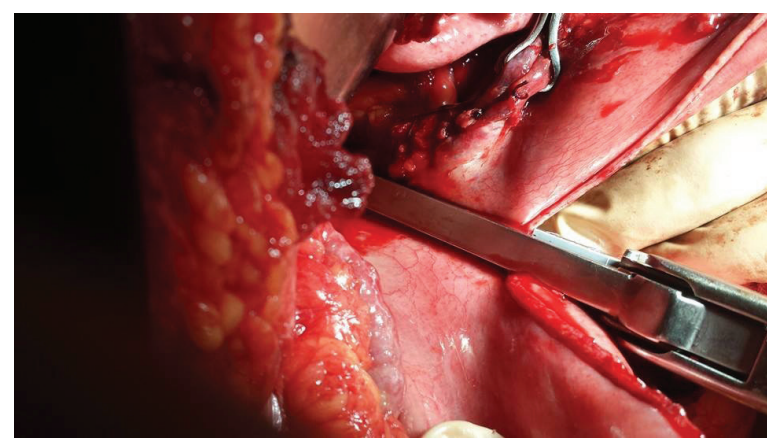

Figure (3): Completion of fundectomy.

in the lesser curvature or cardia.

All patients were hospitalized for either bleeding gastric fundal varices; or previous episodes of variceal bleeding. When we first used this surgical method, most of the patients treated had already experienced failed preoperative intervention and had an uncontrollable hemorrhage or hemodynamic instability from continuous hemorrhage. As we gained clinical experience, we determined that fundectomy and periesophagogastric devascularization was recommended for patients with gastric fundal variceal bleeding at an earlier stage, before their condition deteriorated, regardless of the results of endoscopic intervention. When active bleeding ceased spontaneously and only adherent blood clots were observed at the time of endoscopic diagnosis, fundectomy and periesophagogastric devascularization was undertaken. In emergency cases, the operation was performed without delay.

Each patient was subjected to History taking and thorough clinical examination noting onset of illness, number of previous attacks of bleeding and previous sclerotherapy. Laboratory investigations included $\mathrm{CBC}$, liver functions, renal functions, random

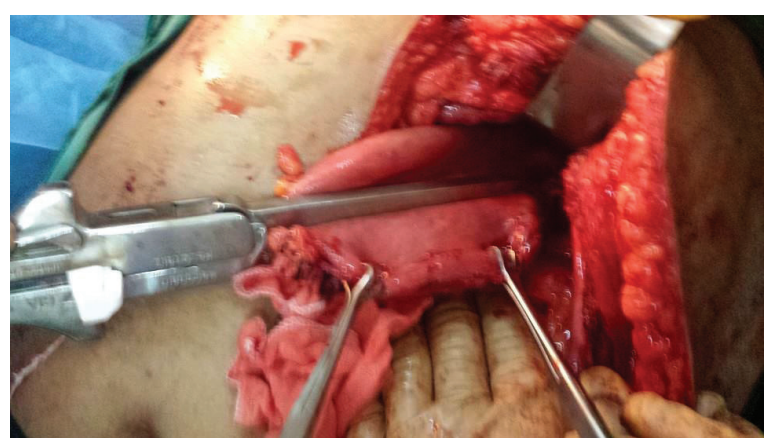

Figure (2): Beginning of fundectomy.

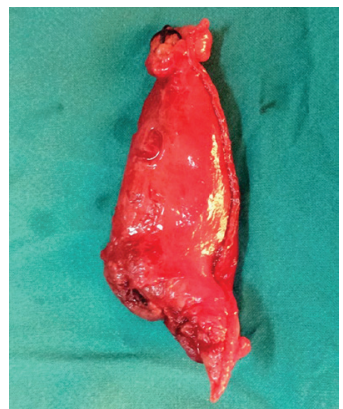

Figure (4): After Excision.

blood sugar, prothrombin time \& activity and hepatitis B \& C markers. Abdominal Ultrasound examination was done. Upper GIT endoscopy was done for determination of the site of varices, classification according to Sarin and sclerotherapy was done when needed.

After doing all the necessary preparation includind blood transfusin if needed, patients were scheduled for operation. Under general anesthesia, through a left subcostal incision, Splenectomy was performed together with periesophageal and perigastric devascularization with left gastric vein and artery ligation at their roots. The site of fundal varices was determined with intraoperative endoscopy, Application of non-crushing curved intestinal clamp until the disappearance of fundal varices was done. After disappearance of fundal varices as noted by the endoscope, resection of the fundus bearing varices at this site of clamping was done using linear stapler for anastomosis. Over sewing of the staple line with prolene or silk 3/0 was done. A drain was inserted and closure of the wound in layers was done.

Post-operatively the following was registered: Postoperative complications like 
Table (1): The characteristics of patients.

\begin{tabular}{|l|l|}
\hline & Patients \\
\hline & $\mathrm{N}: 24$ patients \\
\hline Age & $48.6 \pm 12.6$ years \\
\hline Gender & 18 \\
Males & 18 \\
Females & $6.42 \pm 1.56$ times. \\
\hline Attacks of bleeding & 8 patients $(33.33 \%)$ \\
One attack & 16 patients $(66.67 \%)$ \\
Repeated attacks & 7 \\
2 & 3 \\
3 & 2 \\
4 & 4 \\
5 & 15 patients $(62.50 \%)$ \\
\hline Child-Pugh scoring & 6 patients $(25 \%)$ \\
A5 & 2 patients $(8.33 \%)$ \\
A6 & 1 patient $(8.33 \%)$ \\
B7 & \\
C11 & $11(45.83 \%)$ \\
\hline Causes of liver cirrhosis & $6(25 \%)$ \\
Hepatitis & 14 patients $(58.33 \%)$ \\
Idiopathic & 10 patients $(41.47 \%)$ \\
\hline Sarin's classification & 18 Patients (75\%) \\
Gastric varices limited to the fundus (IGV1). & \\
Gastric varices with esophageal varices (GOV2) & 20 patients $(83.33 \%)$. \\
Portal hypertensive gastropathy. & 2 patients $(8.33 \%)$. \\
\hline Preoperative trials of control of hematemesis & 2 patients $(8.33 \%)$ \\
Endoscopic sclerotherapy or endoscopic variceal ligation & \\
Balloon tamponade. & \\
Failure &
\end{tabular}

Table (2): Postoperative complications.

\begin{tabular}{|l|l|l|}
\hline \multicolumn{1}{|c|}{ Complication } & Number & Percentage \\
\hline Early postoperative morbidity and mortality & 1 & $4.17 \%$ \\
Wound sepsis & 3 & $12.50 \%$ \\
Small subphrenic collection & 2 & $8.33 \%$ \\
Pleural effusion & 2 & $8.33 \%$ \\
Bronchitis & 1 & $4.17 \%$ \\
Portal vein thrombosis & 1 & 4.17 \\
\hline Late Postoperative morbidity & & \\
Delayed gastric emptying & 1 & $4.17 \%$ \\
Incisional hernia & 1 & $4.17 \%$ \\
\hline
\end{tabular}

leakage from the suture line, post-operative wound infection or post-operative liver insufficiency.

Every patient was followed every 2 weeks for 3 months, monthly for 6 months then every 3 months by CBC, liver functions, and morbidity and mortality rates. After 3,6 and 12 months, a follow up upper GIT endoscopy 
Table (3): Factors affecting long-term survival after fundectomy (n: 19), univariate analysis.

\begin{tabular}{|c|c|c|c|}
\hline & $\begin{array}{l}\text { Patients } \\
\text { N: } 24\end{array}$ & $\begin{array}{l}\text { Number of survivors to } \\
\text { end of research N: } 19\end{array}$ & $\mathbf{P}$ \\
\hline $\begin{array}{l}\text { Age } \\
\text { Under } 60 \\
\text { Over } 60\end{array}$ & $\begin{array}{l}20 \\
4\end{array}$ & $\begin{array}{l}18 \\
1\end{array}$ & 0.0029 \\
\hline $\begin{array}{l}\text { Attacks of bleeding } \\
\text { One attack } \\
\text { Repeated attacks }\end{array}$ & $\begin{array}{l}8(33.33 \%) \\
16(66.67 \%) \\
\end{array}$ & $\begin{array}{l}6 \\
13 \\
\end{array}$ & 0.49 \\
\hline $\begin{array}{l}\text { Child-Pugh scoring } \\
\text { A } \\
\text { B and C }\end{array}$ & $\begin{array}{l}21(87.50 \%) \\
3(12.5 \%) \\
\end{array}$ & $\begin{array}{l}18 \\
1 \\
\end{array}$ & 0.59 \\
\hline $\begin{array}{l}\text { Presence of liver cirrhosis } \\
\text { Absence of liver cirrhosis }\end{array}$ & $\begin{array}{l}17(45.83 \%) \\
1(25 \%) \\
\end{array}$ & $\begin{array}{l}13 \\
6 \\
\end{array}$ & 0.014 \\
\hline $\begin{array}{l}\text { Sarin's classification } \\
\text { Gastric varices limited fundus (IGV1) } \\
\text { Gastric, esophageal varices(GOV2) }\end{array}$ & $\begin{array}{l}14(58.33 \%) \\
10(41.47 \%)\end{array}$ & $\begin{array}{l}12 \\
7\end{array}$ & 0.37 \\
\hline $\begin{array}{l}\text { Absence of malignancy } \\
\text { Presence of malignancy }\end{array}$ & $\begin{array}{l}21(87.50 \%) \\
3(12.50 \%)\end{array}$ & $\begin{array}{l}18 \\
1\end{array}$ & 0.038 \\
\hline
\end{tabular}

Table (4): Changes in Child-Pugh scoring of our patients.

\begin{tabular}{|l|l|l|l|l|}
\hline \multicolumn{1}{|c|}{ Child-Pugh scoring } & A5 (patients) & A6 (patients) & B7 (patients) & C11 (patients) \\
\hline Pre-operative & $15(62.50 \%)$ & $6(25 \%)$ & $2(8.33 \%)$ & $1(4.17 \%) \#$ \\
\hline 3 months postoperative & $18(75 \%)$ & $4(16.67 \%)$ & $1(4.17 \%)$ & - \\
\hline 6 months postoperative & $18(75 \%)$ & $4(16.67 \%)$ & $1(4.17 \%)$ & - \\
\hline 12 months postoperative & $17(70.83 \%)$ & $5(20.83 \%)$ & $1(4.17 \%)$ & - \\
\hline
\end{tabular}

\# Patient was deceased on the fifth post-operative day due to hepatocellular failure.

was performed to detect recurrence varices. Patients that did not come for follow up were contacted by telephone. Patients were encouraged to visit the clinic at any time if they have any problem. The mean length of follow up was $49.3 \pm 10.2$ months.

Statistical analysis:

Quantitative variables were expressed as mean \pm SD. Qualitative variables were expressed as frequency and percent. Quantitative parametric variables were compared between the two groups using the unpaired student t-test, quantitative nonparametric variables were compared using Mann-Whitney test. Qualitative variables were compared using Chi-square test or Fisher exact test when the criteria for using Chi-square were not sufficient log-rank tests and Cox's proportional hazards model were used to identify factors influencing long-term survival. The power used was 0.80 while the level of significance was $5 \%$.

\section{Results:}

Patient characteristics:

This study included 18 males (75\%) and 6 females $(25 \%)$. Their age ranged from 28 to 68 years. Mean age was $48.6 \pm 12.6$ years.

Eight patients $(33.33 \%)$ had one attack of bleeding and 16 patients $(66.67 \%)$ suffered from repeated attacks (2-5 attacks). Mean number of attacks of bleeding was $2.42 \pm 1.56$ times.

Fifteen patients (62.50\%) belonged to A5 Child-Pugh scoring, 6 patients $(25 \%)$ were of A6 Child-Pugh scoring, 2 patients (8.33\%) 
were B7 Child-Pugh scoring while the remaining 1 patient $(8.33 \%)$ was $\mathrm{C} 11$ ChildPugh scoring. He was operated due to failure to control an attack of hematemesia by nonoperative means.

According to Sarin's classification, 14 patients $(58.33 \%)$ had gastric varices limited to the fundus (isolated gastric varices type 1, IGV1), 10 patients (41.47\%) had gastric varices concomitant with esophageal varices (gastroesophageal varices type 2, GOV2) and 18 Patients (75\%) had associated portal hypertensive gastropathy.

Of the patients included in the present study, 11 patients $(33.33 \%)$ had positive serum hepatitis $\mathrm{C}$ virus antibody reactivity. No patients had positive serum hepatitis B virus antibody reactivity. History of Bilhariziasis was positive in 3 patients $(12.50 \%)$.

Trying for preoperative control of hematemesis, endoscopic sclerotherapy or endoscopic variceal ligation was attempted and succeeded in 20 patients $(83.33 \%)$. Of the patients in whom the endoscopic procedure failed, 4 patients $(16.67 \%)$ were treated with balloon tamponade. Bleeding was controlled in 2 patients $(8.33 \%)$. In 2 of the 24 patients studied $(8.33 \%)$, operation was performed in the presence of ongoing bleeding Table (1).

Early postoperative morbidity and mortality: One incident of early postoperative mortality occurred during the study. He was the patient with C 11 Child-Pugh scoring who was operated due to failure to control an attack of hematemesia by nonoperative means. He was deceased in the fifth post-operative day due to hepatocellular failure.

Seven patients $(29.16 \%)$ experienced one or more early postoperative complication: 3 had wound sepsis, 2 had small subphrenic collection, 2 had pleural effusion, 1 had bronchitis, and 1 had portal vein thrombosis. All were treated medically and improved.

Late Postoperative morbidity: One patient developed delayed gastric emptying. He gradually improved with prokinetic drugs and food regulation. One patient developed incisional hernia. $\mathrm{He}$ benefited from hernioplasty. Table (2).
Long-term results and survival: There were no bleeding episodes from gastric varices along the follow up period and no recurrences of gastric varices at endoscopy. Three patients with GOV 2 had 1- 3 attacks of hematemesis from esophageal varices starting 13 months after surgery. Bleeding was successfully controlled by endoscopic sclerotherapy.

During follow up 3 patients developed malignancies: 2 hepatocellular carcinomas and 1 uterine cancer. Four patients died of hepatic failure, 2 of them were the 2 who developed hepatocellular carcinomas. No patient died as a result of recurrent variceal bleeding. By univariate analysis, age, Child Pugh scoring and absence of malignancy were significantly associated with survival Table (3).

Endoscopic follow up: All gastric fundal varices disappeared completely with no recurrence. In $6(25 \%)$ out of 10 patients, esophageal varices disappeared completely. Three patients $(12.50 \%)$ downgraded to grades I and II. One patient $(4.17 \%)$ remained with grades III and IV. Endoscopic follow up schedules were done. Endoscopic sclerotherapy was done once bleeding developed. There was improvement in portal hypertensive gastropathy.

Laboratory follow up: Downgrading of Child-Pugh scoring of patients was noticed Table (4). Mean hemoglobin level at the time of admission was $7.99 \pm 1.35 \mathrm{~g} / \mathrm{dL}$. Patients received from 3 to 5 units of fresh blood during preoperative preparation. No blood transfusion was needed during operation except for the patient operated during active bleeding. By the third month of follow up, mean hemoglobin level reached $11.90 \pm 0.89$ $\mathrm{g} / \mathrm{dL}$ ( $\mathrm{p}$ less than 0.0001).

\section{Discussion:}

There are many nonoperative treatment options for the bleeding gastric varices, such asendoscopic sclerotherapy, placement of transjugular intrahepatic portosystemic shunt (TIPS) and balloon-occluded retrograde transvenous obliteration (B-RTO). However, controlling hemorrhage and preventing 
recurrent hemorrhage with these methods has been far from satisfactory, and operative intervention is often inevitable. ${ }^{14-19}$

We previously proposed that fundectomy and periesophagogastric devascularization can be considered as a means of controlling gastric fundal variceal bleeding and of preventing recurrent hemorrhage. ${ }^{12,20}$ After gaining experience with this modality, we considered adding intra-operative endoscopy to guide accurate localization and complete eradication of varices and to minimize the resected area of the gastric fundus. This procedure decreased the morbidity compared to our previous researches not using the intra operative endoscopy.

Periesophagogastric devascularization with esophageal transection, as described by Sugiura and Futagawa, has been widely used to treat gastric variceal bleeding, particularly in Japan. ${ }^{21}$ This procedure does not necessarily require esophageal transection, which was devised to prevent recurrent esophageal hemorrhage in patients with gastric variceal bleeding. Furthermore, if esophageal variceal bleeding recurs, endoscopic intervention can be used for effective control. Esophageal transection strongly contributes to postoperative morbidity and mortality, especially in patients that have undergone extensive sclerotherapy. ${ }^{22-24}$

Devascularization of periesophagogastric vessels, as reported by Hassab and associates, is also widely used to treat gastric variceal bleeding. ${ }^{11,25,26}$ However, gastric varices remain visible in the intramural area after this technique, and they might cause recurrent gastric variceal bleeding. Some investigators have reported that proximal gastrectomy can eradicate varices-bearing lesions and thereby reduce recurrent bleeding rates. ${ }^{27,28}$ However, additional anastomosis between the esophagus and distal stomach is necessary, a procedure that could lead to life-threatening complications, particularly in patients who have undergone previous esophageal sclerotherapy.

The operative method described in the present study is relatively straightforward: It involves excision of the part of fundus carrying fundal varices and simultaneous peri-esophagogastric devascularization to block blood inflow. With this procedure no operative mortality occurred. This improvement may have been related both to improved patient conditions at operation, because operative intervention was decided earlier. Inspite of the overall improvement in the mortality rate, the operative mortality among patients with Child-Pugh classification $\mathrm{C}$ or ongoing bleeding remained high, which is comparable with the findings of other studies. ${ }^{22,23}$ Recognizing that surgical intervention is inevitable for patients with uncontrollable variceal bleeding, it should be noted that in the present study around $50 \%$ of these high-risk patients with uncontrollable hemorrhage survived after fundectomy and periesophagogastric devascularization.

Patients who experience more attacks of bleeding episodes may achieve a better survival than those with fewer bleeding episodes. Although it is possible that patients who have survived previous episodes have a better overall condition and better liver function, their survival might also be related to the perceived urgency of their disease, which may have reduced the time from bleeding onset to hospitalization. Also, patients with a prior bleeding history probably are better at recognizing early symptoms, such as, melena or hematochezia, and are much better informed about the significance of bleeding signs. It is therefore important that patients with cirrhosis receive adequate information about the early signs and symptoms of variceal bleeding.

Even after periesophagogastric devascularization, a hypertensive and congestive state exists in the portal system and the possibility of recurrent variceal bleeding remains (its rate has been reported to range from $5 \%$ to $40 \%$ ). ${ }^{29}$ Moreover, because esophageal variceal bleeding can be successfully controlled by endoscopic intervention or conservative treatment, the majority of deaths resulting from the hemorrhagic complications of cirrhosis are probably attributable to gastric varices. ${ }^{26}$ Thus, the minimization of recurrent gastric variceal 
bleeding should be the primary surgical goal. In the present study, no recurrent bleeding occurred from gastric varices over a median follow-up of $49.3 \pm 10.2$ months. However, recurrent esophageal variceal bleeding did occur in 3 patients, and it was successfully controlled by endoscopic intervention.

In general, about one-third of patients with advanced liver cirrhosis die of uncontrollable variceal bleeding. Akiyoshi et al. Reported an overall 5-year survival rate of $34 \%$ and concluded that fundal variceal bleeding, hepato-cellular carcinoma, and hepatic functional reserve are prognostic factors of long-term survival. ${ }^{30}$ Both hepatocellular carcinoma and Child-Pugh classification were found to be significant prognostic factors of long-term survival.

The present study shows that fundectomy and periesophagogastric devascularization can prevent variceal bleeding with its consequences, and it demonstrates that the prevention of recurrent gastric variceal bleeding should be considered a crucial treatment for cirrhotic patients with this condition.

\section{Conclusion:}

\section{Gastric} fundectomy and periesophagogastric devascularization is associated with a low incidence of recurrent bleeding and offers good long-term survival. It is an effective therapeutic option in patients with fundal variceal bleeding. Intraoperative endoscopy helps to guide accurate localization and complete eradication of varices and to minimize the resected area of the gastric fundus.

\section{Reference:}

1- Lee JH, Han HS, Kim HA, et al: Long-term results of fundectomy and periesophgogastric devascularization in patients with gastric fundal variceal bleeding. World Journal of Surgery 2009; 33, 2144-2149.

2- Nevens F, Broeckaert L, Rutageerts P, et al: The long-term morbidity and mortality in a cohort of patients with liver cirrhosis and esophageal varices. Hepatogastroenterology Journal 1995; 42, 979-984.

3- Sarin SK, Lahoti D, Saxena SP, et al:
Prevalence, classificationand natural history of gastric varices: A long-term follow up study in 568 portal hypertension patients, Hepatology Journal 1992; 16, 1343-1349.

4- Chin KJ, Korula J, Yamada S, et al: Demonstration of two distinct subsets of gastric varices. Digestive Disease and Sciences 1991; 36, 303-309.

5- Trudeau W, Prindiville T: Endoscopic injection sclerosis in bleeding gastric varices. Journal of Gastrointestinal Endoscopy 1986; 32, 264-268.

6- Kanagawa H, Mima S, Kouyama H, et al: Treatment of gastric fundal varices by balloon-occluded retrograde transvenous obliteration. Journal of Gastroenterology and Hepatology 1996; 11: 51-58.

7- Azoulay D, Castaing D, Majno P, et al: Salvage transjugular intrahepatic portosystemic shunt for uncontrolled variceal bleeding in patients with decompensated cirrhosis. Journal of Hepatology 2001, 35, 590-597.

8- Tripathi D, Therapondos G, Jackson E, et al: The role of the trans-jugular intrahepatic porto-systemic stent shunt (TIPSS) in the management of bleeding gastric varices: Clinical and hemodynamic correlations. Gut J 2002; 51: 270-274.

9- Tomikawa M, Hashizume M, Saku M, et al: Effectiveness of gastric devascularization and splenectomy for patients with gastric varices. Journal of the American College of Surgeons 2000; 191: 498-503.

10- Luketic VA: Management of portal hypertension after variceal hemorrhage. Clinics in Liver Disease Journal 2001; 5: 677-707.

11- Han HS, Yi NJ, Kim YW, et al: New operative method for fundal variceal bleeding: Fundectomy with periesophagogastric devascularization. World Journal of Surgery 2004, 28: 406-410.

12- El-Gengehy M, Nagy A, Enaba M: Evaluation of fundal minigasterectomy for the management of isolated fundal varices. MSC Thesis, faculty of Medicine, Tanta University 2007; 102.

13- Dib N, Oberti F, Cales P: Current management of the complications of portal hypertension: Variceal bleeding and ascites. Canadian Medical Association Journal 2006; 174: 1433-1443.

14- Kojima K, Imazu H, Matsumura M, et al: Sclerotherapy for gastric fundal variceal bleeding: Is complete obliteration possible without cyanoacrylate? $J$ Gastroenterol 
Hepatol 2005; 20: 1701-1706.

15- Taniai N, Yoshida H, Mamada $\mathrm{Y}$, et al: The treatment of gastric fundal varicesendoscopic therapy versus interventional radiology. Hepatogastroenterology 2005; 52: 949-953.

16- Arai H, Abe T, Shimoda R et al: Emergency balloon-occluded retrograde transvenous obliteration for gastric varices. $J$ Gastroenterol 2005; 40: 964-971.

17- Noophun P, Kongkam P, Gonlachanvit S, et al: Bleeding gastric varices: Results of endoscopic injection with cyanoacrylate at King Chulalongkorn Memorial Hospital. World J Gastroenterol 2005; 11: 7531-7535.

18- Enne M, Pacheco-Moreira LF, Balbi E et al: Transjugular intrahepatic portosystemic shunt versus $\mathrm{H}$-graft portacaval shunt in the management of bleeding varices: A cost-benefit analysis. Ann Surg 2006; 243: 139-140.

19- Yoon CJ, Chung JW, Park JH: Transjugular intrahepatic portosystemic shunt for acute variceal bleeding in patients with viral liver cirrhosis: Predictors of early mortality. AJR Am J Roentgenol 2005; 185: 885-889.

20- Othman I, Enaba M: Stapled versus manual fundal minigastrectomy with periesophagogastric devascularization in the treatment of bleeding fundal varices. Tanta Medical Sciences Journal 2006; 1(3): 103-114.

21- Sugiura M, Futagawa S: A new technique for treating esophageal varices. $J$ Thorac Cardiovasc Surg 1973; 66: 677-685.

22- Jin G, Rikkers LF: Transabdominal esophagogastric devascularization as treatment for variceal hemorrhage. Surgery 1996; 120: 641-649.

23- Gouge TH, Ranson JH: Esophageal transection and para-esophagogastric devascularization for bleeding esophageal varices. Am J Surg 1986; 151: 47-54.

24- Dagenais M, Langer B, Taylor BR et al: Experience with radical esophagogastric devascularization procedures (Sugiura) for variceal bleeding outside Japan. World $J$ Surg 1994; 18: 222-228.

25- Nakamura H, Goseki N, Dobashi $\mathrm{Y}$ et al: Hassab operation with intraoperative endoscopic injection sclerotherapy "HassabEIS" for esophagogastric varices: With an autopsied case after excessive gastric vascular damage. Hepatogastroenterology 1996; 43: 980-986.

26- Hassab MA: Gastroesophageal decongestion and splenectomy. A method of prevention and treatment of bleeding from esophageal varices associated with bilharzial hepatic fibrosis: Preliminary report. $J$ Int Coll Surg 1964; 41: 232-248.

27- Hsieh JS, Huang CJ, Huang TJ: Management of isolated gastric varices by devascularization and proximal gastrectomy in cirrhotic patients. HPB Surg 1994; 7: 201-209.

28- Orloff MJ, Orloff MS, Daily PO, et al: Longterm results of radical esophagogastrectomy for bleeding varices due to un-shuntable extrahepatic portal hypertension. Am J Surg 1994; 167: 96-103.

29- Henderson JM: Surgical treatment of portal hypertension. Baillieres Best Pract Res Clin Gastroenterol 2000; 14: 911-925.

30- Akiyoshi N, Shijo H, Iida T et al (2000) The natural history and prognostic factors in patients with cirrhosis and gastric fundal varices without prior bleeding. Hepatol Res 2000; 17: 145-155. 


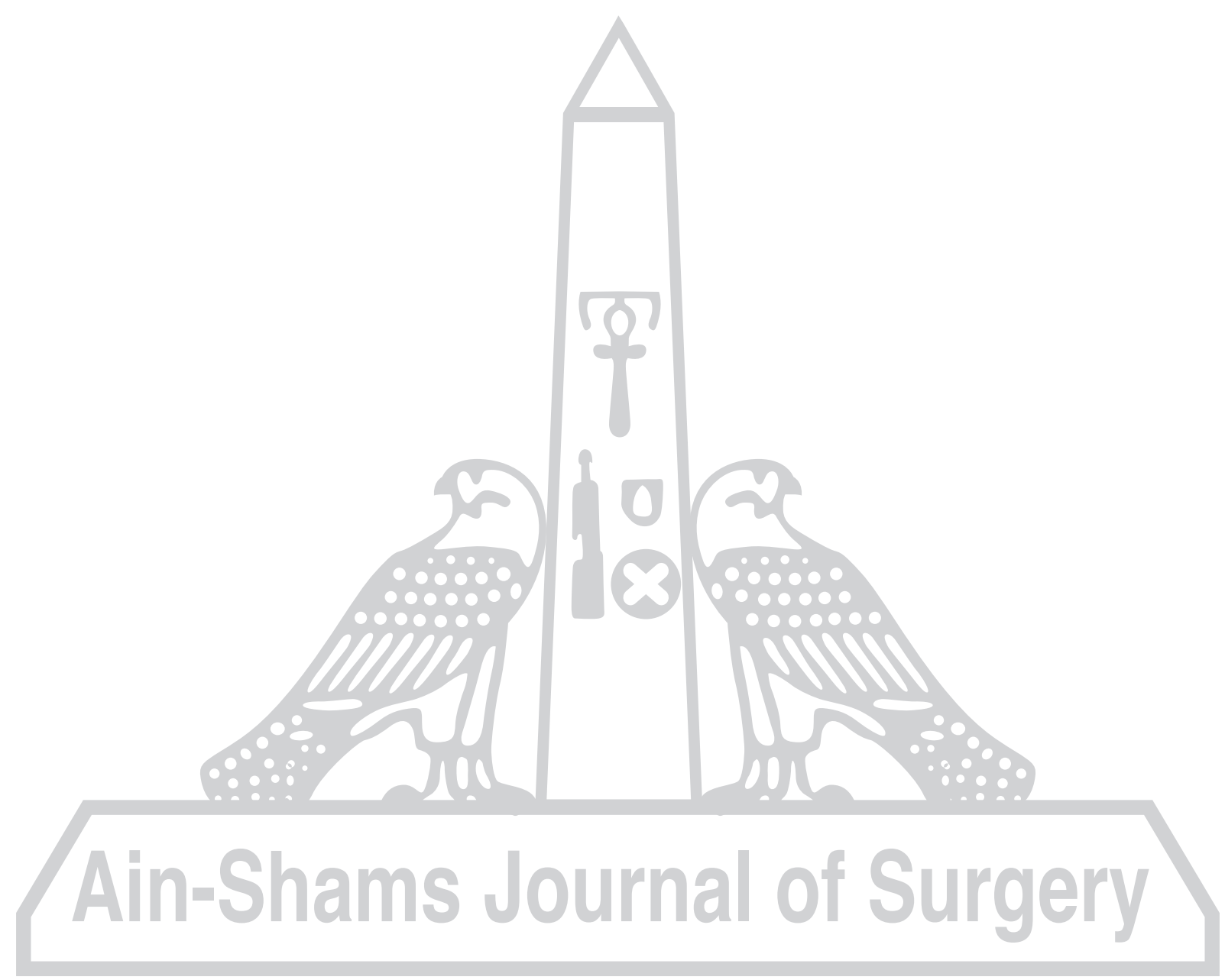

